\title{
Amelia fu Marion: «I me you the others». Appunti per il recupero degli scritti inglesi di Amelia Rosselli
}

Amelia of Marion: "I me you the others". Notes on a Re-reading of Amelia Rosselli's English Writings

\section{Silvia Mondardini}

\section{OpenEdition}

\section{Journals}

Edizione digitale

URL: http://journals.openedition.org/cei/1285

DOI: $10.4000 /$ cei. 1285

ISSN: 2260-779X

\section{Editore}

UGA Éditions/Université Grenoble Alpes

\section{Edizione cartacea}

Data di pubblicazione: 30 juin 2013

Paginazione: 281-302

ISBN: 978-2-84310-245-5

ISSN: 1770-9571

\section{Notizia bibliografica digitale}

Silvia Mondardini, «Amelia fu Marion: «I me you the others». Appunti per il recupero degli scritti inglesi di Amelia Rosselli», Cahiers d'études italiennes [Online], 16 | 2013, online dal 15 décembre 2014 consultato il 26 mars 2021. URL: http://journals.openedition.org/cei/1285 ; DOI: https://doi.org/ 10.4000/cei.1285 


\section{AMELIA FU MARION: «I ME YOU THE OTHERS». APPUNTI PER IL RECUPERO DEGLI SCRITTI INGLESI \\ DI AMELIA ROSSELLI}

Silvia Mondardini

Università di Bologna

\section{Le ragioni di una lettura gender-oriented}

Amelia Rosselli (Parigi 1930 - Roma 1996), uno dei nomi più interessanti della letteratura italiana del Novecento, una delle poche presenze femminili fortemente riconosciute a livello istituzionale, ormai canonizzata anche dalla sua assunzione tra i Meridiani della Mondadori, è stata letta a lungo «in nome del Padre», come «figlia di soli padri»: qui vorrei accennare a una ipotesi di riscrittura della storia nel nome della Madre, andando oltre un pattern interpretativo classico e forse limitante, ormai abbandonato dalla critica più recente ${ }^{\mathrm{I}}$. Mantenendosi al di qua di quella «sovrapposizione pseudofemminista e pseudopsicologica» che la Rosselli

I. Va segnalato che già Pasolini (Pier Paolo Pasolini, Notizia su Amelia Rosselli, «Il Menabò», giugno I963, poi anche in Amelia Rosselli, Variazioni belliche, Roma, Fondazione Piazzolla, 1995, pp. 8-Io) inseriva nella sua analisi sulla Rosselli i riferimenti ai miti decadenti della Nevrosi, del Mistero e dell'Irrazionale, che sono proprio quelli con i quali la cultura occidentale ha spesso identificato il femminile. Tra le primi indagini in questa direzione si veda poi Nelson Moe, At the Margins of Dominion: The Poetry of Amelia Rosselli («Italica», 69, 2, Summer 1992, pp. I77-I95), dove il titolo del saggio dell'intellettuale americano è una citazione tratta dal commento pasoliniano e dove si sottolinea come la singolarità dell'opera di Amelia Rosselli risieda proprio nella sua perspicace analisi dei rapporti di potere operanti nella contemporanea società capitalista, convincente proprio in virtù della sua posizione triplicemente anomala derivante dalla sua formazione straniera, inglese e francese prima che italiana, dal suo essere studiosa di musicologia oltre che letterata e — non ultimo dall'essere donna in un contesto culturale per lo più maschile. Utile anche Mario Moroni, La presenza complessa: identità e soggettività nelle poetiche del Novecento, Ravenna, Longo, 1998, che dedica un interessante capitolo al soggetto poetico femminile con particolare riferimento alla poesia rosselliana. Su questa linea voglio citare almeno l'acuto saggio di Tatiana Bisanti, La poesia di Amelia Rosselli: il silenzio disturbato, la comunicazione assoluta, in Scrittura femminile, Italienische Autorinnen in 20. Jahrhundert zwischen Historie, Fiktion und Autobiographie, a cura di Irmgard Scharold, Tübingen, Gunter Narr Verlag, 2002, pp. 26I-280 e la più recente monografia di Emmanuela Tandello, Amelia Rosselli, La fanciulla e l'Infinito (Roma, Donzelli, 2007), che esamina le figure femminili presenti nell'opera rosselliana. Per ogni ulteriore lettura, nonché per il rinvio agli

Cahiers d'études italiennes, $\mathrm{n}^{\circ} \mathrm{I} 6,20 \mathrm{I} 3$, p. 28I-302. $28 \mathrm{I}$ 
ha avuto modo di avversare in molti suoi interventi ${ }^{2}$, credo che il confronto con la lingua della Madre, un inglese non sussunto con il latte materno ma 'succhiato' dai libri, sia un buon punto d'avvio per capire l'Amelia $f u$ Marion piuttosto che l'Amelia fu Carlo. Del resto è noto che il legame che Amelia Rosselli intrattenne con il femminismo è ambivalente, probabilmente molto più articolato e sfumato di quanto in genere fino a oggi si è voluto indicare. Per quanto in alcuni saggi e interviste la poetessa faccia notare a più riprese quelli che, secondo lei, sono i difetti di tanta poesia femminista o scritta da donne ${ }^{3}$, ovvero il suo carattere intimistico e autobiografico, è necessario non cadere nella tentazione d'interpretare certe affermazioni come spia di un totale rifiuto: un interesse vivo e diretto della poetessa verso la questione femminile è esistito ed è concretamente documentato dall'esistenza di un gruppo non trascurabile di libri conservati nella sua biblioteca personale ${ }^{4}$.

Una lettura al femminile è legittimata da due aspetti: innanzitutto tra le carte del Fondo di Pavia (recentemente pubblicate da Chiara Carpita) si leggono le pagine scritte nel 1953 durante il periodo di cura con Bernhard, psicoanalista di scuola junghiana, dove Amelia appuntava tra i problemi emersi, per lei essenziali da risolversi, quello del «senso di inferiorità: complesso forse comune a una grande parte delle donne, motivato in questo caso specialmente dalla rivalità con i fratelli; rivalità per l'affetto della

sviluppi critici più recenti, il riferimento è a Daniela La Penna, "Cercatemi e fuoriuscite": Biography, Textuality, and Gender in Recent Criticism on Amelia Rosselli, «Italian Studies», 65, 2, July 20I0, pp. 278-285.

2. Amelia Rosselli, Istinto di morte e istinto di piacere, "Nuovi argomenti», 67-68, luglio-dicembre 1980, pp. I75-178. Si veda anche Mariella Bettarini, Per un'intervista inedita ad Amelia Rosselli, in Amelia Rosselli. Un'apolide alla ricerca de linguaggio universale, a cura di Stefano Giovannuzzi, Atti della giornata di studio, Firenze, Gabinetto Vieusseux, 29 maggio 1998, "Quaderni del Circolo Rosselli», 17, Firenze, Giunti, I999, pp. 82-86: «Il grosso difetto della letteratura femminile o un pochino femminista oggi è quello di essere orgogliosa. È la pecca numero uno del femminismo letterario. Io ho letto insieme a delle femministe dei libri [...], non sanno uscire dalla loro vita privata. [...] la poesia [...] se non sublimano, non esiste.[...] Se il rapporto diventa plurale, si può parlare di un discorso ad un pubblico, se non è al plurale, tanto vale non farlo.»

3. Aggiungo qui anche il rinvio a Paola Febbraro, Amelia Rosselli: Lezioni e conversazioni (in Daniela Attanasio ed Emmanuela Tandello, Amelia Rosselli, "Galleria», 48, I-2, gennaio-agosto 1997, p. 20I) e Aurelio Andreoli, $\grave{E}$ possibile far poesia al femminile? Intervista ad Amelia Rosselli ("Paese sera», 28 agosto 1980).

4. Tra i libri della sua biblioteca: Toni Wolff, Structural Forms of the Feminine Psyche (privately printed for the students), Zürich, Association C. G. Jung Institute, July 1956; Luigi De Marchi, Sociologia del sesso, Bari, Laterza, 1963; Reimut Reiche, Sessualità e lotta di classe, Bari, Laterza, 1969; Carla Lonzi, Sputiamo su Hegel, Milano, Scritti di rivolta femminile, I970; Kate Millet, Prostituzione. Quartetto per voci femminili, Torino, Einaudi, I975; Wilhelm Reich, The Function of the Orgasm, Sex-Economic Problems of Biological Energy, London, A Panther Book, 1979; Lorenzo Braibanti e Paride Braibanti, Nascere meglio, Roma, Editori Riuniti, I980; Adele Cambria, Il Lenin delle donne, dalla castrazione amorosa alla violenza terrorista, (contributi di Nilde Jotti, Rossana Rossanda, Clara Valenziano, Cecilia Castellani), Padova, Mastrogiacomo, Images 70, I98I; Dalle donne la forma delle donne, Carta itinerante, idee, proposte, interrogativi. Documento a cura della sezione femminile della direzione del PCI, Roma, Botteghe Oscure, 1986; Patrizia Violi, L'infinito singolare: considerazioni sulla differenza sessuale nel linguaggio, Verona, Essedue, 1986. 
madre, e rivalità intellettuale». La Rosselli sottolineava — in ultimo — «il problema di uscire dalla "torre d'avorio" nell'arte, e contemporaneamente nei rapporti umani, sessuali»:

È anche qui che l'atteggiamento cosciente, derivato intellettualmente in gran parte da libri, si dimostra molto in avanti della realtà psichica interna. Si è troppo identificati all'animus nella sua forma delle facoltà spirituali e pensanti [nella sua forma spirituale, volontaria, e non si prende affatto in considerazione quello che è invece il processo di maturazione tipico della donna: graduale, vegetale, collegato alla terra. Viene cercata un'ipercompensazione della facoltà pensante] [...].5

Significativamente la Rosselli usa il termine animus e non anima, laddove è proprio Jung a usare il termine latino anima per l'Archetipo del femminile la cui esperienza è riservata all'uomo, mentre l'animus è la figura corrispondente nell'inconscio della donna, comunque figura che comunica le immagini dell'inconscio alla coscienza, in un'essenziale funzione di mediazione, cruciale per quel processo di decentramento della personalità dall'Io conscio al Sé che è lo scopo dell'analisi junghiana. La Rosselli parla consapevolmente come una donna, in una linea che può fare pensare — ad esempio - alla Zambrano, che si dichiarava convinta che per essere realmente 'vivente' la filosofia avrebbe dovuto rompere l'egemonia della mente per farsi carico dell'essere umano nella sua interezza, esplorando «il logos che scorre nelle viscere» ${ }^{6}$. L'attenzione femminile alla physis, comune ad Amelia Rosselli, rinvia anche all'intuizione zambraniana della ragione poetica: oltre la modalità di conoscenza tipicamente occidentale caratterizzata da "uno sguardo disattento alle cose», María contrappone un cammino di conoscenza nel quale l'oggettività non è distacco neutro ma fiduciosa apertura al reale, «innocenza originaria, verginità dell'anima» ${ }^{7}$. La donna-poeta Rosselli è perfettamente in linea con queste intuizioni.

A ciò si aggiunga il secondo aspetto che veicola questa lettura genderoriented: il vuoto della Madre è stato un dato importante quanto la perdita del Padre. Amelia dice: "Quella di mia madre è stata una perdita molto brutta, più brutta di quella di mio padre perché, quando è morto

5. Chiara Carpita, Tre scritti e un acquerello per Ernst Bernhard, in La furia dei venti contrari, Variazioni di Amelia Rosselli. Con testi inediti e dispersi dell'autrice, a cura di Rosaria Lo Russo e Stella Savino, Firenze, Le Lettere, 2008, p. I30. Le parentesi quadre indicano una sezione del testo presente solo in uno (S.3a) dei due testi inediti parzialmente coincidenti presi in considerazione dall'autrice (sigla d'archivio S.3 e S.3a).

6. María Zambrano, citata da Elena Laurenzi in All'ombra del dio sconosciuto: Antigone, Eloisa, Diotima, a cura e con traduzione di Elena Laurenzi, Milano, Nuove Pratiche Editrice, 1997, p. 19. Su questo tema vedi María Zambrano, Filosofia e poesia, a cura di Pina De Luca, Bologna, Edizioni Pendragon, 1988, che porta in appendice un'utile bibliografia sia dei lavori di Zambrano che della letteratura critica relativa.

7. María Zambrano, Verso un sapere dell'anima, Milano, Raffaello Cortina, 1996, p. 94. 
mio padre, ero troppo piccola per sentire un dolore adulto.» ${ }^{8}$ Aldo Rosselli è convinto che Amelia «avrebbe dato qualsiasi cosa per essere lei, per averla» ${ }^{9}$. Rocco Scotellaro ricorda: "Quando morì la madre le andarono in bocca le parole della lingua della fanciullezza.» ${ }^{\text {10 }}$

Nel mondo inglese della mia infanzia cadeva

la monade. La monade sorvegliava riccamente

il mondo. ${ }^{\text {II }}$

La madre-monade fu — di fatto - una figura cruciale e la donna - la donna come concetto - nella sua formazione, è virile e attiva. Per la nonna, Amelia Pincherle Rosselli, punto di riferimento cruciale per la nipote, l'attività della donna doveva comprendere anche un impegno intellettuale e professionale per l'affermazione di sér2. La figura di questa donna nuova, nonna Amelia l'aveva messa in scena in un'opera teatrale di grande successo, Anima: criticando la donna superficiale, vacua e frivola, qui impersonata da Graziana, le contrapponeva una donna vigorosa e sincera, Olga, donna indipendente come l'Emma di Illusione o le 'eroine' di El rèfolo e di El socio de papà. Lei stessa, peraltro, fu consigliera e - poi - vicepresidente del Consiglio Italiano delle Donne che «raccoglieva una vera élite femminile come intelligenza e spirito d'iniziativa» ${ }^{13}$.

8. Maria Pia Ammirati, Amelia Rosselli. Improvvisi/lapsus, in Madamina: il catalogo è questo, Messina, Rubbettino, 1995, p. I08.

9. Fotobiografia: conversazione con Aldo Rosselli, in Dossier Amelia Rosselli, a cura di Siriana Sgavicchia, "Il Caffe Illustrato", I3-I4, luglio-ottobre 2003.

Io. Rocco Scotellaro, Un lago nella memoria, «Trasparenze», numero monografico a cura di Giorgio Devoto ed Emmanuela Tandello, I7-I9, 2003, pp. 8I-82.

II. Amelia Rosselli, Le poesie, a cura di Emmanuela Tandello, prefazione di Giovanni Giudici, Milano, Garzanti, 1997, p. 250: di seguito si indicheranno con la sigla PS i Primi scritti inclusi nell'edizione.

I2. La nonna della poetessa, Amelia Pincherle Rosselli (Venezia I870 - Firenze 1954), dopo essersi separata dal marito nel 1903, allevò da sola i suoi tre figli: Aldo, Carlo e Nello Rosselli. Era piuttosto nota ai suoi contemporanei come scrittrice di teatro e tra le sue opere si possono ricordare il dramma Anima (Torino, Lattes, I90I) di ascendenza ibseniana e le commedie in dialetto veneziano El rèfolo e El socio de papà. Scrisse anche alcune fiabe per bambini. Cominciò la propria collaborazione con la rivista fiorentina «il Marzocco» nel 1904 con un articolo intitolato Discussioni sul femminismo. Nel novembre del i913 pubblicò sullo stesso giornale l'articolo Propaganda elettorale femminile pronunciandosi in favore del suffragio femminile. Quando Carlo e Nello furono assassinati nel 1937, assieme alle nuore e ai nipoti, iniziò una serie di soggiorni attraverso l'Europa per sfuggire al fascismo e alla minaccia delle leggi razziali: arrivò con la famiglia in America nel 1940 dove restò fino alla fine della guerra. Durante l'esilio americano partecipò alla Women's Division della Mazzini Society. Nel 1945 pubblico l'articolo Strade vecchie e strade nuove, in «Uguaglianza!» (rivista del Movimento Femminile del Partito d'Azione), partecipando al dibattito ancora in corso sul diritto di voto delle donne. Per approfondimenti si veda il volume Amelia Rosselli, Memorie. I ricordi della mamma dei fratelli Rosselli, a cura di Marina Calloni, Bologna, Il Mulino, 20or, e Amelia Pincherle Rosselli, a cura di Vieri Dolara, "Quaderni del Circolo Rosselli», 3, 2006.

13. Maria Bianca Viviani Della Robbia, Ricordo di Amelia Rosselli, in Ricordo di Amelia Pincherle Rosselli nel cinquantesimo anniversario della sua scomparsa, Atti del Convegno organizzato dal Lyceum Club Internazionale di Firenze e dal Circolo di Cultura politica «Fratelli Rosselli» (4 dicembre 2004), a cura di Patrizia Bulletti, Riccardo Pratesi e Mirka Sandiford, Firenze, Alinea, 2006. 
Amelia - pur vicina alle prime rivendicazioni femministe - era già oltre: viveva sola dai diciotto anni, aveva ricevuto un'educazione aperta a diverse letterature e diverse religioni e soprattutto era stata educata da sempre all'insegna della giustizia e della libertà ${ }^{14}$. Del resto la Rosselli dichiarò: «Se il movimento femminista si propone una piattaforma socio-politica sono anche d'accordo, ma resta da studiare un'altra piattaforma forse, quella appunto biologico-culturale (o perfino mistica), che non è del tutto da buttare via.» ${ }^{15}$

\section{Trame psicoanalitiche negli scritti inglesi}

Con queste premesse, per leggere l'Amelia fu Marion mi sono concentrata sulle opere scritte nella lingua della madre, dunque sulla sua produzione inglese ${ }^{16}$, la meno indagata a oggi. L'inglese letterario, che era anche la lingua di una madre assente, è linguisticamente altrettanto importante che l'italiano del padre da lei canonizzato: tuttavia, dopo avere tradotto l'intero corpus rosselliano, inediti inclusi, sono convinta che non ci sia «lingualatte» e che la specificità della Rosselli sia che la sua lingua madre è il corpus della tradizione. L'inglese è, sì, territorio femminile/materno come apertura a «spazi del sentimento», come la nostra accenna in alcuni inediti fogli manoscritti su Joyce (che ho rinvenuto tra le sue carte al «Fondo Rosselli» di Pavia) ${ }^{17}$, ma è intellettuale come lingua, una lingua che è

\footnotetext{
I4. Giustizia e Libertà fu un movimento politico liberal-socialista fondato a Parigi nell'agosto del I929 da un gruppo di esuli antifascisti, tra cui il padre di Amelia, Carlo Rosselli.

15. Amelia Rosselli, in Donne in poesia, a cura di Biancamaria Frabotta, Roma, Savelli, 1976.

16. My clothes to the wind (PS 5-9), October Elizabethans (ottobre 1956) (PS 55-67), A birth (PS I23-I29), in Sleep. Poesie in inglese, a cura di Emmanuela Tandello, Milano, Garzanti, 1992 (si indica qui e di seguito con la sigla SL seguita dal numero della pagina). Per tutti i testi citati da Sleep le traduzioni sono della curatrice del volume garzantiano, per i testi inediti le traduzioni sono mie.

17. Al "Fondo manoscritti» del Centro di ricerca sulla tradizione manoscritta di autori moderni e contemporanei dell'Università di Pavia il materiale relativo alla produzione inglese di Amelia Rosselli è raccolto in cinque cartelle, la maggior parte del materiale è costituita da fogli dattiloscritti, meticolosamente fotocopiati più volte dalla stessa Rosselli e minuziosamente ricontrollati per inserire i dati tecnici circa la pubblicazione in riviste, nonché il numero delle righe di cui si compone ogni testo, che è riportato a mano su ognuno dei fogli riprodotti. Nello specifico SL.I è la cartella di riferimento per tutte le citazioni successive: contiene una copia dattiloscritta di Sleep che riporta l'annotazione per mano della Rosselli «copia con appunti pubblicazioni» e l'intestazione interna "Amelia Rosselli, Lungotevere Sanzio 5, Roma, Italy» e include anche quelle poesie che sono state escluse e non inserite nell'edizione Garzanti del 1992 e che qui riportano l'indicazione manoscritta a matita "escluso non tradotto"; sui margini dei fogli ci sono indicazioni a matita delle sedi di pubblicazione, a volte dettagliate e a volte più sbrigative; in calce a ogni poesia c'è l'indicazione dell'anno di stesura delle singole poesie. Gli inediti che cito vengono riportati con il numero relativo alla loro posizione tra i testi segnalati con la dicitura «escluso non tradotto»: al momento della consultazione (aprile 20IO) i materiali non erano ancora stati riordinati in modo definitivo e i fogli relativi a Joyce a cui ci si riferisce qui erano sparsi all'interno della cartelletta in questione.
} 
acquisita, una lingua che le è più familiare nelle modulazioni dei padri Donne, Shakespeare, Joyce, Cummings and company che nel suono vocale di Marion. "The empty mother» - il vuoto della madre — del giovanile My Clothes to The Wind (PS 5-9) racconta della madre assenza e della fuga dalla sua impossibile patria, la matria Inghilterra: il «latte» che nutre, Amelia lo trova nei libri e nelle voci dei padri della letteratura.

Anche se la voce che domina il ductus poetico rosselliano è trina e non c'è lingualatte effettiva, va rilevato - tuttavia - che se l'italiano è sempre rivisto entro una griglia metrica e il francese rivisitato in chiave surrealista, l'inglese si mantiene in una sfera dove l'acustica e il suono possono avere inciso con maggiore libertà. Amelia, sprovvista di una qualsivoglia lingua imposta, mobile tra le lingue, si muove su percorsi affini proprio a quelli di Cixous $^{18}$, e il fulcro della sua scrittura sta nel ritrovare proprio la lingualatte che si diceva, la lingua-canto materna, la lingua agrammaticale che affiora nella grammaticalità della lingua scritta, una lingua che fluisce liberamente e si fa materia, corpo narrante: la specificità della produzione rosselliana in lingua inglese sta in questo, in questo essere spesso oltre il significato.

Del resto il nitore ineccepibile dell'immediato è precluso a ogni lingua e l'unico modo di scrivere in maniera immediata è quello di adottare una grammatica che risponda alla logica del sistema inconscio. Il tentativo della Rosselli è stato quello di riconvertire questo linguaggio plurale in una grande parola piena: l'utilizzo del linguaggio dell'altro è stato una forma di ricerca del «tempo perduto», nel senso di rinvio a una dimensione ontologicamente precedente a quella storica. Ciò è tanto più vero per la produzione nella lingua della parte femminile della sua famiglia: se la psicoanalisi ci insegna che l'acquisizione di un'identità personale passa attraverso un processo di individuazione dell' Io che si avvia nel confronto col linguaggio corporeo e verbale della madre, sembra che questo percorso per Amelia si avvii solo attraverso la poesia degli anni Cinquanta e Sessanta. Pare quasi che il mancato rapporto con la madre possa essere ricostituito attraverso questo rincorrerla nella lingua che fu sua, in una rincorsa che cerca di recuperare anche quel mancato processo di individuazione, quel tragitto di costituzione del Sé mai propriamente concluso per quel mancato contatto emotivo con la lingua materna. Amelia, che come ricorda la cugina Silvia non parlava bene nessuna lingua e manteneva un marcato accento straniero (straniero in ogni lingua), usa l'inglese

I8. Per ogni riferimento alla Cixous, si veda Hélène Cixous, Entre l'écriture, Paris, Des Femmes, I986 (a p. 32 parla di «languelait») e Il teatro del cuore, Parma, Pratiche, I992 (edizione originale 1990). 
per scelta, si getta nella babele del proprio inconscio polipolide alla ricerca di quel primo contatto con il Séris.

Le stesse prose inglesi incluse in Primi scritti sono dei percorsi onirici alla ricerca di sé: $A$ birth (PS I25) inizia con una frase ripresa cinque volte in variatio, "If I lay back layers of time», poi «If I lay back on layers of time» e, se in My clothes to the wind (PS 9) si termina con l'approdo momentaneo e onirico "to the recognition", nel secondo caso - come ci si chiarisce in chiusura - si tratta di un effettivo «sample of recognition", dunque un campione o un modello di autoanalisi, che nel termine inglese include una sorta di sfumatura ufficiale di 'riconoscimento' quasi giuridico. La stessa Rosselli disse che, se fu influenzata «dal neorealismo e dal marxismo", tanto da non perdere «di vista nemmeno per un istante l'esperienza», se voleva distaccarsene, scriveva in inglese, "mille miglia sopra la realtà italiana ${ }^{20}$. Trattenne a lungo dalla pubblicazione i suoi versi inglesi, quasi una reticenza a salvaguardia di un serbatoio testuale che si voleva lontano dallo spazio pubblico firmato nel nome del padre "Amelia Rosselli» ${ }^{2 \mathrm{r}}$ : a questi testi Amelia riserva l'indicibile più impronunciabile, quello che seguendo Lacan potremmo chiamare registro dell'Immaginario $^{22}$. Va detto che gli albori dello scrivere inglese della nostra si ascrivono — da subito - a questo regime onirico-immaginario: Marina Calloni ha raccolto tre poesie degli anni 1948-1949, autografe, in inglese, ritrovate nell'archivio di famiglia, Lullaby, Circuit e The Vase ${ }^{23}$ : se l'ultima parla di una mano che scivola in delle cavità e la seconda di una «morning dissipation", la prima prelude al sonno più adulto della raccolta Sleep. Le phantasies scritte in seguito alla terapia sono in inglese e dissotterrano dal tesoro dei ricordi le immagini intraviste molto tempo addietro.

"I me you the others» "2: le figure emergono con più facilità quando la Rosselli scrive in inglese, che è anche la lingua della terapia. Il lessico junghiano, le parole — o i «motivi» — dello psicanalista svizzero sono fortemente presenti nel suo repertorio lessicale. Il «mandala», termine sanscrito

19. Sulla lingua materna si rinvia a Allinizio di tutto. La lingua materna, a cura di Eva-Maria Thüne, Torino, Rosenberg \& Sellier, 1998 e Jacqueline Amati-Mehler, Simona Argentieri e Jorge Canestri, La babele dell'inconscio. Lingua madre e lingue straniere nella dimensione psicoanalitica, Milano, Raffaello Cortina, I990.

20. Silvio Perrella, Per Amelia Rosselli, «Nuovi argomenti», I2, I997, pp. I2-I3.

2I. Intervista ad Amelia Rosselli, a cura di Marco Caporali, "Poesia», aprile I990, p. 9.

22. Si vedano almeno Jacques Lacan, "Dei Nomi-del-Padre» seguito da "Il trionfo della religione», Torino, Einaudi, 2006 e Materia e senso. Pratiche significanti e teoria del linguaggio, Torino, Einaudi, I980 (edizione originale 1980).

23. Citato in Maria Calloni, Le due Amelie e le diaspore della lingua m/paterna, in «Se/dalle tue labbra uscisse la verità». Amelia Rosselli a dieci anni dalla scomparsa, Atti del Convegno del Circolo Rosselli dell'8-9 giugno 2006, a cura di Stefano Giovannuzzi, Firenze, Giunti, "Quaderni del Circolo Rosselli, 3", 2007, p. 30.

24. «I me you the others» è il verso iniziale del trentaquattresimo testo inedito conservato al «Fondo» di Pavia. 
che letteralmente unisce "essenza» e "possedere, contenere» e che viene tradotto con "cerchio", figura geometrica effettivamente derivata dal cerchio incrociato con il quadrato, atta alla delimitazione dello spazio entro il quale si relazionano i vari piani di realtà, è strumento di meditazione, contemplazione e ascesi, ed è designato con diverse figure, tutte «intese da Jung come immagini del Sé attraverso le quali viene all'espressione la totalità psichica, a partire dalla condizione di dispersione in cui si trova il soggetto ${ }^{25}$. Queste stesse figure tramano gran parte della produzione di Amelia Rosselli, che - consapevole - scrive di un «muro» che è «immagine mentale di sé stesso» (inedito 27) e che "la rosa e la rugiada/ non sono che immagini di te; piccoli / simboli, sulla gran ruota» (SL 28).

La Rosselli, oltre alle tante presenze di occhi, ulteriori forme previste dal mandala junghiano, scrive di una terra fatta "quasi tonda» (SL 8), di una terra di forma tonda (SL 68), di una terra che "rounded the point» — «'accerchio», traduce la Tandello, «rotondò il punto», direi io- (SL 96), di un «round world» (SL I84), di una terra che accerchia «nuda/ il punto di disperazione», di un deviare «attorno al punto posato / appunto sul quadrato che perturba questo / mio tondo corpo", di qualcuno che "ha doppiato la punta [rounded the point] posando/ quadrati così nudi sulla tonda superficie» (SL 204). Non è altro che la «tirannia d'immagini» lamentata in uno dei testi più tardi di Sleep (SL IO2). Non può essere un caso: la Rosselli era psicoanaliticamente troppo consapevole perché lo possa essere. Quali dei mandala, i testi rosselliani — soprattutto gli inediti, tali proprio per la loro difficoltà — sono visioni provenienti dall'inconscio, estranee alla logica e al senso del conscio: penso all'inedito dove si chiudono «tutte le imposte» e ci si precipita nel buio delirio della propria mente (inedito I6) o alle "esplosioni creative» che "alla fine arrivano» (inedito I8).

Il processo è simile a quello che si compie nel percorso dalla nigredo alchemica, per assurgere alla rubedo ultima: del resto già nel Diario in tre lingue del 1955-1956 Amelia aveva scritto:

mi domando se non sia possibile, in un assurdo sforzo, dimenticare quanto è intorno a me per rinchiudermi nelle alchimie di un linguaggio buono ad ogni latitudine. (PS 95)

La Rosselli butta dentro all'athanor, la fornace, il proprio reale per ottenere la pietra filosofale. La logica entro cui rileggere le figurazioni rosselliane potrebbe più agilmente essere quella cinese, quella che oltrepassa i concetti causalistici occidentali e domina l'I Ching, Il Libro dei 
Mutamenti ${ }^{26}$, ulteriore 'altro' rosselliano. A campione prendo il numero ventisette dei sessantaquattro segni essenziali, l'«I», «Gli Angoli della bocca (il sostentamento)», che "è l'immagine di una bocca aperta; sopra e sotto le labbra salde, e in mezzo l'apertura della bocca»: è la "hoary cavern mouth» (SL 20), sono le "wide open mouths» di un inedito (inedito 35), limitandosi ad alcune delle undici occorrenze del termine, a cui andrebbero affiancate le cinque occorrenze di «lips». Ancora più agile è il rinvio all' esagramma uno, "Ch'ien», "Il Creativo", simboleggiato anche dal cavallo, principio maschile ma proteiforme immagine della poetessa in molte delle sue poesie. Se nel Diario la Nostra scrive "generalmente pratico la Ritirata» (PS 72), "Tun - La Ritirata» è l'esagramma trentatré, se scrive che «Il Mite/ Obey with Holiness / humility and gentleness toward your Self, and God» (PS II7), ancora una volta dietro si può rintracciare un esagramma, il cinquantasette, «Sun - Il Mite (il Penetrante, il Vento)». Le linee costituenti i trigrammi e gli esagrammi simboleggiano elementi essenziali, che sono anche disseminati nella poesia del mutamento della Rosselli: cielo, terra, tuono, acqua, monte, vento, fuoco e lago. Del resto è Amelia, gravemente dipendente dal libro oracolare cinese, a rivelare la traccia già nel suo Glossarietto esplicativo, laddove in merito alla frase «seguire/ il servizio d'un re», segnala "frase tipica dall'I King, libro divinatorio pre-taoista cinese)»: il King si ripresenta ben venticinque volte negli scritti inglesi ed è - probabilmente - il medesimo «re» al cui «servizio» è in Variazioni belliche (VB 237, 275 e 276). Le diciannove occorrenze dell'espressione "as if» sono ugualmente significative e - anche per queste - una chiave di lettura è Jung, che insegna che «ogni interpretazione rimane necessariamente al "come se..."”, dal momento che «l'ultimo nucleo di significato può essere circoscritto, non descritto» ${ }^{27}$. Lidea — junghianamente intesa — è immagine del modello delle cose (del loro archetipo) come pure delle cose stesse nella loro rappresentazione mentale o psichica: tra l'ipotesi dell'idea come ciò che fa essere le cose essendone essenza e quella dell'idea come mero segno rispetto al quale le cose hanno un carattere primario, si impone una contrapposizione formativa. Sono due inediti - in questo caso — che confermano anche questo sostrato:

26. I King: il libro dei mutamenti, traduzione italiana dalla versione tedesca di Richard Wilhelm a cura di Bruno Veneziani e Gaetano Ferrara, confrontata con l'originale cinese da Bruno Veneziani, Roma, Astrolabio, I950 (Viterbo, Fondo Rosselli).

27. Carl Gustav Jung e Kàroly Kerényi, Prolegomeni allo studio scientifico della mitologia, Torino, Bollati Boringhieri, 1999 (edizione originale 1942), p. II6. 
An idea is a host, embarrassed it might resign its post. ${ }^{28}$

An idea is a host, embarrassed [sic] it might

have to resign its post: a post is a

hot tower, lying in the midst, no, peeking

through high clouds. [...]

Your idea is a host, selling screams for

bewitchery, breaking those clouds through

with triumphant haste, taste of the battle. ${ }^{29}$

Un'idea-ostia, spirito e carne, essenza e cosa insieme. Lo stesso concetto della morte (termine ricorrente ben venti volte nel corpus inglese) ha evidenti ascendenze junghiane: Jung è la filigrana teorica del «would it were death» di Amelia, del suo timore della «rossa onda / del vero vivere» (VB I63), della sua ansia di separazione da tutto e brama di non bramare, di arrivare «al punto in cui tutto il desiderio/ muore» (SL 47).

Così come la traiettoria di un proiettile termina al bersaglio, la vita termina nella morte, che è quindi il bersaglio, lo scopo di tutta la vita. ${ }^{30}$

\section{La rilegatrice di anima e corpo: cenni a Virginia Woolf e Dylan Thomas}

Se il primo 'altro' che si incontra leggendo la Rosselli inglese alla rincorsa della madre è l'Es, le tante altre voci sono poi i tanti altri spettri che spesso si rimaterializzano nei suoi versi.

Leggendo la poesia rosselliana si giunge facilmente alla conclusione che Amelia vive una difficile e pesante iper-coscienza del suo fare letteratura, una estenuante consapevolezza della risemantizzazione della parola poetica che si stratifica eternamente senza mai potersi sigillare in qualcosa di definitivo e rassicurante: mi sembra che Amelia sia invischiata 'a morte' nel circolo vizioso della letteratura. Ci si trova in una situazione di intertestualità massiccia che rende impossibile la pretesa di uno schema classificatorio delle varie voci 'tra le righe': la vocazione intertestuale della nostra ha una forte e costante valenza bellica che - nel confronto-acquisizione — riduce tutto a pezzi: per questa autrice, la mis-lettura non è sempre un gioco ma una cosa estremamente seria.

28. Inedito $2 \mathrm{I}$.

29. Inedito 3I.

30. Carl Gustav Jung, La dimensione dell'inconscio, in Opere complete, vol. Io, Torino, Bollati Boringhieri, I992 (edizione originale 1967), p. 438. 
Del resto anche dai suoi brevi scritti critici si ricava sempre come minimo comune denominatore l'essenzialità dell'autenticità della scrittura, la preminenza data alla capacità degli autori recensiti di aderire alla propria ispirazione, presupposto senza il quale non si ha vera letteratura: l'autenticità per Amelia Rosselli sta in quelle forme di scrittura dall'impronta fortemente personale, come quella di Pasternak, poeta che «non chiarisce il mistero della sua fecondissima originalità, del suo tastare ogni terreno ideologico con inaspettata libertà, singolarità» ${ }^{3 \mathrm{I}}$, come quella dei versi «limpidi e raggianti» di un Sergio Solmi, capace di una poetica "molto personalmente sua» ${ }^{32}$. Al di là di una lettura che sfidi Amelia nella composizione di un catalogo di reperti citazionistici, alla rincorsa di filiazioni e corredi genetici, anche di marca femminile, cosa assai ardua e perniciosa, proporrei invece una lettura che cerchi di collocarla all'interno di una categoria, evidenziando ulteriori consonanze all'interno del mondo della letteratura, al quale appartiene ma entro il quale continua a essere un elemento straniero e in continua mobilità. In quest'ottica Sleep è un exemplum di un confronto con la tradizione, peraltro una tradizione quanto mai aperta, che si potrebbe sciogliere rinvenendo le modalità di rilettura, sempre in un'ottica di linee d'onda, alla ricerca di similitudini del pensiero o di involontari rinvii ${ }^{33}$.

Per sistematizzare un'indagine del genere a posteriori credo si potrebbero raccogliere delle apparizioni in 'figure': Demetra, Diotima, Alice e Penelope (dietro ci sta - ovviamente - un noto studio di Adriana Cavarero) ${ }^{34}$. Lungo questa linea, sincronicamente e diacronicamnte, esistono contiguità ideali tra donne nella storia: l'aspetto performativo della teatralizzazione del Sé, che ama e/o che soffre, l'ironia che gioca sul role play destabilizzante dell'Io e del Tu, il martirio del corpo e della lingua. Quest'ultimo aspetto è quello che vorrei qui accennare e che mi piacerebbe simbolicamente riconnettere alla figura di Penelope: la tessitrice che ri-lega l'anima e il corpo separati dalla filosofia degli uomini è la poetessa Amelia che ri-lega parole attente alla corporeità o - meglio — che sono la sua corporeità: «Hell, loomed out/ with perfect hands», ovvero «l'inferno, tessuto/ da mani perfette» (SL IO-II).

31. Amelia Rosselli, La fatica di essere autentico, in Una scrittura plurale, a cura di Francesca Caputo, Novara, Interlinea, 2004, p. I30.

32. Amelia Rosselli, Dal balcone e L'angelo attento, in Una scrittura plurale, cit., pp. 89-90.

33. Visitando il Fondo di Viterbo, dove è conservato quanto rimane di ciò che era nella biblioteca dell'ultima casa romana della nostra, la sensazione è quella di una casa infestata di spettri: prendere tra le mani i suoi libri, pieni di sottolineature e appunti, rovinati e vissuti, aiuta a capire la vastità delle letture della poetessa e la profondità del suo approccio.

34. Adriana Cavarero, Nonostante Platone: figure femminili nella filosofia antica, Roma, Editori Riuniti, 1999. 
Se la madre è il «vuoto» delle prime prose, il padre è il «senso di non corporeità» di cui parlò la poetessa in un'intervista ${ }^{35}$ : l'evanescenza è la marca dei rapporti famigliari di Amelia Rosselli e la violazione dell'involucro corporeo è l'operazione propedeutica alla (ri)acquisizione della realtà. Del resto, «una madre indifferente è una madre che impedisce la differenziazione» ${ }^{36}$ e la soluzione può essere quella di fare tutto a brandelli, nel tentativo di dominare come una Amazzone, sempre pronta a combattere per difendersi. Un corpo a corpo con le cose, con i corpi, con la realtà interiore ed esteriore.

Osmosi o dominio. Evanescenza del corporeo o ricerca di un corpo.

Del resto la Rosselli, pur convinta che «sul piano linguistico [non] si riveli la femminilità», aveva dichiarato che «la donna con la sua fisiologicità corporale $[\ldots]$ ha qualcosa non di diverso da scrivere, ma di più fisiologico da distinguere anche sul piano contenutistico» ${ }^{37}$.

L'attenzione al corpo è attenzione all'oggettività delle 'cose' — anche delle 'cose della lingua' - e interesse per la corporeità effettiva: in quest'ultimo senso, quello che sembra si possa rilevare nella poesia della Rosselli è la presenza di un corpo androgino, la progressiva sublimazione della sessualità che passa attraverso la tessitura del proprio soggetto nella scrittura (il «noi» generico della poesia d'apertura di Sleep stupra la «sister navel» tradotta come "fratello ombelico»). Nelle Note a Sleep per l'edizione Rossi \& Spera del 1989, l'autrice aveva commentato proprio questa poesia («Well, so, patience») come "dalla passione sessuale alla sublimazione nello scrivere», istituendo il corpo come punto di partenza. Il corpo in scena è un corpo e basta: la carne in torsione e spesso torturata non è - tuttavia asessuata, ma bisessuale. "Allora, negli anni 6I-63, si parlava molto del corpo femminile che doveva entrare nell'arte, ma anche il corpo maschile doveva entrare nell'arte, nella parola, nella poesia, nella filosofia, non c'è differenza.» ${ }^{38}$ Oltre certe limitanti basse canonizzazioni maschili e al di là delle rivendicazioni femminili, il corpo permane come problema metafisico, materia femmineo-maschile che non riesce a trascendere la sua forma e rimane chiuso in una cella.

35. Giacinto Spagnoletti, Intervista ad Amelia Rosselli, in Una scrittura plurale, cit., p. 299.

36. Didier Anzieu, Il pensare. Dall'io-pelle all'io-pensante, Roma, Borla, 1996, p. 67 (edizione originale 1994).

37. Amelia Rosselli, in La furia dei venti contrari, cit., p. 73.

38. Gabriela Caramore, Paesaggio con figure, programma RAI Radio 2; ora in È vostra la vita che ho perso. Conversazioni e interviste 1964-I995, a cura di Monica Venturini e Silvia De March, Firenze, Le Lettere, 20IO, pp. 265-319. Il tema del corpo non è stato esclusivo appannaggio del movimento delle donne, ma — è in questa sede la stessa Rosselli a ricordarlo — dalla fine degli anni Cinquanta e soprattutto negli anni Sessanta, era diventato centrale in tutta l'arte e la letteratura come "strategia di resistenza dell'umano», da Pasolini a Pagliarani, da Zanzotto a Sanguineti e a Volponi. 
Nella copia rosselliana del Pensiero selvaggio di Lévi-Strauss, Gian Maria Annovi ${ }^{39}$ ha ritrovato un ritaglio di giornale - con annotazioni autografe di Amelia - di un articolo di Brunetto Chiarelli apparso su "Paese Sera» il 5 novembre 1978 e intitolato I nostri antenati senza coppie fisse: lo cito a conferma di un interesse della Rosselli per l'ipotesi di una pansessualità oltre le distinzioni di genere. Del resto, in quel "poemetto politico, femminista oltre che poetico" ${ }^{40}$ che è La libellula ${ }^{4 \mathrm{I}}$, si rintraccia un'androginia diffusa e in un inedito Amelia scrive e traduce:

Paul was in his socket all day long and longed for Divinity, for she was getting socket-thin and the lust for fever was an ever tempting thing.

Paul era nella sua cavità tutta la giornata e fortemente desiderava la Divinità, perché diveniva cavo-magro e il desiderio di febbre era una perenne tentazione. ${ }^{42}$

Amelia rende il «she» senza esplicitarlo, ma poi prosegue traducendo «diveniva cavo-magro» e sembra seguire una lingua transgender che rende un soggetto maschile, in linea con il Paul d'apertura ma che rinnega il «she», un supposto soggetto femminile sulla scena che scompare: una netta ambiguità sessuale che il traduttore potrebbe essere tentato di sciogliere leggendo il Paul «nella sua propria cavità» e la «lei», "cava-magra» come una scoperta allusione a un rapporto dove il soggetto maschile è dominante. Il senso assoluto dell'altro non è dato con una marca sessuale stabile, e questo rende difficile il processo di individuazione dell'Io attraverso il Tu. Il rinvio è all'Orlando woolfiano, dove credo si racconti la più grandiosa storia di androginia di tutti i tempi: l'ambiguità sessuale appare come una precondizione della scrittura, secondo un'ottica ampiamente sviluppata proprio dalla Woolf. In lei un'entità fissa non è mai data, per cui è impossibile essere una cosa oppure esserne un'altra: si è in uno stato di continuo assemblaggio e successiva dissolvenza, come la sua Mrs. Dalloway che, mentre cammina per le strade di Londra, si disperde nell'aria mattutina. «She would not say of Peter, she would not say of

39. Si veda Gian Maria Annovi, Time can stop (and it does): un inedito da Sleep, in La furia dei venti contrari, cit., pp. I89-190.

40. Amelia Rosselli e Marina Camboni, Incontro con Amelia Rosselli, «DonnaWomanFemme», I, 29, gennaiomarzo I996, p. 7I.

4I. Amelia Rosselli, La libellula, Milano, Studio Editoriale, 1985.

42. Inedito 8, presentato con la traduzione della stessa Amelia Rosselli presente in foglio manoscritto siglato SL.Id presente nella cartella SLi del Fondo Rosselli di Pavia. 
herself, I am this, I am that», ma si trova fusa in date forme e colori in dati momenti. Anche la concezione unitaria del corpo scompare: «this body she wore... this body, with all its capacities, seemed nothing - nothing at all.» ${ }^{43}$ The Waves - poi - è integralmente percorso dall'ossessione per la dissoluzione del corpo. Il 28 maggio 1929 la scrittrice riflette sulla ricerca del suo personaggio, che non deve essere «a Lavinia or a Penelope»: «I want "She".» ${ }^{44}$ Queste immagini pre-simboliche rinviano a quello che la Kristeva ha concettualizzato come l'antitesi del simbolico, il semiotico. Pensa Rhoda: «But here I am nobody. I have no face.» ${ }^{45} \mathrm{Ma}$ il simbolico è ineludibile: «I have to bang my hand against some hard door to call myself back to the body.» ${ }^{46}$

Nella Rosselli si replica il refrain «we are three» (SL I22, SL I50, SL 206), si moltiplicano in variatio lacerti di testi che presentano soggetti differenti, domina la difficoltà della resa dei pronomi, perché il soggetto recitante è almeno trino: «I me you the others», ripete in un inedito (inedito 34).

You would not take responsibility [sic]: you would not wipe the hinge clean, you would not be a body [...]. (SL 200)

Impera il dramma dell'identità, la messa in scena della scissione di un io che sussiste solo in una dimensione frattale: il soggetto «invece di trascendersi in una finalità o in un insieme che lo supera, si diffrange in una moltitudine di ego miniaturizzati, tutti simili gli uni agli altri» ${ }^{47}$. $\grave{E}$ in questo modo che nelle poesie rosselliane ci si muove tra «I», «We» e «They», tutte identità-in-scena, tutte il medesimo soggetto e ugualmente un soggetto diverso: tutte parodia dell'identità, carnevalizzazione del supposto «Io». Si tratta di una caratteristica significativamente presente in molti testi femminili, tanto che si è potuto parlare di una sorta di coscienza plurima, come fa Sheila Rowbotham in un'analisi che si concentra sulla scrittura di Hilda Dolittle ${ }^{48}$. Tutto è scomposto, s-corporato: scrittura inclusa. Il quadro della Lily di To the Lighthouse della Woolf è figura del testo stesso e il suo problema di «how to connect this mass on the right hand with that on the left» non è che la difficoltà di trovare una mediazione tra i termini

43. Virginia Woolf, Mrs Dalloway, Oxford, Oxford University Press, 20oo, p. 8.

44. Virginia Woolf, The Diary of Virginia Woolf, 5 voll., a cura di Anne Olivier Bell, London, The Hogarth Press, 1977-I984, pp. 229-30.

45. Virginia Woolf, The Waves, London, Collector's Library, 2005, p. 28.

46. Ibid., p. 37 .

47. Jean Baudrillard, L'altro visto da sé, Genova, Costa \& Nolan, 1997 (edizione originale 1987).

48. Si veda Susan Stanford Friedman, Penelope's Web: Gender, Modernity, H.D.'s Fiction, Cambridge, Cambridge University Press, 1990, p. 85. 
rigidamente chiusi dell'opposizione binaria. In Sleep si legge: «Ed ora che l'ora di posa è finita / il tuo nuovo principio ti fissa / negli occhi, e con terrore/ sospetta che mai/ fosti nata prima di scrivere/ di teneri sospetti» (SL I46): anche il quadro di Lily, come la poesia in posa per un ritratto di Amelia, è un tentativo di riportare tutto a unità.

Una pluripersonalità — secondo la nota intuizione di Auerbach ${ }^{49}$ — è in scena nell'episodio woolfiano del "calzerotto marrone», presente sempre in To the Lighthouse. C'è un inedito rosselliano (inedito I6) che dissemina tra i versi un «sock» che non può che sembrare epifania del «reddishbrown hairy stocking» del quale si occupa proprio Mrs. Ramsay: Amelia parla di «vane apostrofi» perché tutte le imposte vengano chiuse, si parla di un calzino che - apparentemente insignificante - salta fuori da un cassetto, di mondanità e sopraffazione. La filigrana sembra quella del testo di Virginia, nel quale Mrs. Ramsay nota le porte aperte e si chiede indispettita perché nessuno si ricordi di chiuderle, mentre lavora a maglia un calzino da portare al figlio del guardiano del faro che hanno intenzione di visitare l'indomani, ma che, sopraffatti dal caso, non visiteranno che anni dopo. Il calzerotto dell'una e dell'altra non sono importanti in sé ma lo sono per quello che provocano: non conta la loro realtà obiettiva ma il loro riflesso interiore. L'inedito rosselliano in questione è un sibillino «frangersi della coscienza», difficile da sciogliere e quasi impossibile da tradurre: il corpo della realtà e il corpo della scrittura vengono passati al setaccio dell'anima e della coscienza interiore e s-corporati.

La scrittura appare per Amelia e Virginia una vibrazione, un tremore della mente che cerca la verità delle cose, percepibile quando queste sfuggono, mutanti. I corpi delle cose entrano in contatto nel loro trasmutarsi: il gruppo dei sei di The Waves chiarisce che la singola cosa, il singolo corpo, si esalta nello strabordare dalla confinazione nel proprio, nell'incrocio che mina la stabilità e sospinge verso le possibilità dell'anomalia. Peraltro «waves» compare come unicum rosselliano solo nell'inedito che citavo sopra e che appare woolfiano anche nel suo chiudersi accennando a una vita che «inexistent flutter/ on, a-brest of wings». L'onda definisce la curvatura che la realtà fisica e corporea della Woolf e della Rosselli sceglie di accettare. Il corpo non è alternativa al pensiero, bensì una sua forma: attraverso la corporeità passa la conoscenza, anche secondo l'antico principio del "pathei mathos». Amelia — come già Emily Dickinson e Sylvia Plath, tanto amate e lette — violenta le sue poesie: nel Diario in tre lingue

49. Si veda Erich Auerbach, Il calzerotto marrone, in Mimesis. Il realismo nella letteratura occidentale, vol. II, Torino, Einaudi, 1999 (edizione originale 1946), pp. 305 ss. 
scriveva che «un buon metodo di domazione [sic] è quello di affrontare la ferocia prima che essa si difenda (arrivi a difendersi)» (PS IOI). Diceva la Dickinson: "The Attar from the Rose [...] is the gift of Screws.»"

Non si ha tempo di incontrare questa Madame de Sade di Trastevere ${ }^{\text {II }}$, ma voglio qui accennare anche alla valenza di questo corpo/mente di Amelia. Se il richiamo alla corporalità è costitutivo della poesia italiana degli anni Settanta, in Amelia Rosselli assume una significatività massima e vuole «contrapporre la matericità del percepire, la forza incarnata della parola, a una violenza omologante camuffata da normalità» ${ }^{5^{2}}$.

Una parola cruciale del mondo inglese rosselliano è parola corporale: «belly». Sono persuasa di un vincolo mentale tra "cuore» e «ventre» nella Rosselli. Amelia parla di «her heart/ [come] a belly» (SL I66), invoca lo «sweet heart, [e di seguito un] belly-ache» (SL 172), e così a seguire. Tra le carte inedite, si rintraccia poi «the waist's belly-boat» (37), una fitta trama di presenze in un testo che recita "time is no belly», «belly's charm», «I've had enough: of / this belligerence», «terrified bellies» (35), e l'occorrenza in un inedito cruciale (3I), che chiarisce la «belligeranza» come il nocciolo della questione.
Here lies
A.R. — she sits, lies, stands, drinks twists her mind out of order then puts it back again into order, with a thin- lipped smile, a jaunt on her new face and a triangular smirk on her fat hips rounding the point, rounding the point! of belligerency.

La belli-gerenza — ciò che 'si porta dentro' - è sempre connessa con la necessità d'amare, con l'inevitabilità del morire e con l'essenzialità dello scrivere, temi portanti di tutti i testi dove ricorra la parola tematica «belly», variamente declinata ${ }^{53}$. Laddove invoca il «dolce, dolce, dolce, bimbo", prosegue rivolgendosi a un "grembogravido rifiuto" (che io preferisco all'ipotesi tandelliana di "bellicoso spreco»), una "penetrante delicatezza», una «saliva che osserva/ il mistero della morte che è una penetrazione»: il finale è un appello alle cose che «ti prendono per mano/ e supplicano:

50. Emily Dickinson, Tutte le poesie, a cura e con un saggio di Marisa Bulgheroni, Milano, Mondadori, 1997 (trad. it. di Marisa Bulgheroni, con in appendice traduzioni di Amelia Rosselli, pp. I660-I667), n. 675.

5I. Nel 1954 la Rosselli si trasferì definitivamente in Italia, a Roma, acquistando una casa a Trastevere, dove rimase fino al I97I.

52. Niva Lorenzini, La poesia italiana del Novecento, Bologna, Il Mulino, 1999, pp. I64-165.

53. Si vedano ad esempio SL 154-155, I66, I73, I79, I87. 
esprimimi!» (SL I54) Del resto le viscere da cui viene la voce sono anche la pancia che può - femminilmente — generare: si pensi — appunto - al neologismo "bellygerence», che vincola — anche foneticamente — all'idea di gravidanza quanto a quella di lotta. Dalla pancia al resto. Le stesse «mani», le «tender heavy fat hands» (SL 8) dell'ouverture tornano nei due versi che compongono il secondo componimento che definisce un'atmosfera infernale («hell, loomed out/ with perfect hands», SL Io): seguono «il nostro collo nudo abbrividito», la «nostra mano nuda», la «nostra sorella ombelico» che bisogna imparare a stuprare, le «dita spezzate gelide» di Otello, la ruota di tortura nella poesia della «rosa e la rugiada», lo «stendardo insanguinato», la «lebbra» che la assale, gli esseri umani che si «trascinano terreni», «earth bound», il «cibo corporale» nel "pacco incartato», il Re e la regina «decapitati» e $\mathrm{i}$ "loro spasmodici sensi», la bocca che «si torce/ sconnessa», gli «ingranaggi» della mente che «stridono sino ad uno stop stridente», il «ramoscello del tuo corpo» che «sopporta tutto, / ruotando attorno al suo stesso spiedo», il "cuore tubercoloso», «la mandibola penzola» e ancora ventri, schiene, mani e dita.

Del resto, sulla sua copia dei Pensieri di Leonardo da Vinci, la Rosselli aveva sottolineato con un pastello alcuni versi: «FACCIÀNO NOSTRA VITA COLL'ALTRUI MORTE» ${ }^{54}$. La questione cruciale sta nella materia e «matter», foneticamente vincolato all'italiano «materia» (e i suoni delle parole per la poetessa sono spesso veicolo di sensi trasversali) ${ }^{55}$, è termine che - nel corpus inglese - torna ben ventidue volte e due nella forma plurale: la nostra parla di mani che «do but cut a slice into the heart/ of the matter» (SL 80) ${ }^{56}$ e altrove scrive che "a revolution / in the heart was no matter, if you had your / mind on things» (inedito I5). Il nocciolo della questione per la Rosselli sta nell'impossibilità di una reale comprensione reciproca, la questione è che la materia ci rende irrimediabilmente distanti. La poetessa è «una briciola che non coagula» (PS 5), detrito nato dalla corrosione della materia matrice, qualcosa di consunto e destinato alla deriva.

Quest'Amelia così attenta al corpo e al corpo delle parole per molti aspetti può ricordare anche Dylan Thomas ${ }^{57}$ : la carne è alla base della

54. Leonardo da Vinci, Pensieri, in Tutti gli scritti, a cura di Augusto Marinoni, Milano, Rizzoli, I952, p. 59 (Viterbo, Fondo Rosselli). I maiuscoli sono nel testo.

55. Si veda l'esempio del Diario in tre lingue (I955-1956), in PS 70-I22.

56. Forse tra le righe ci potrebbe essere anche un «altro», il Graham Greene di The Heart of the Matter del I948, volume presente nella Biblioteca rosselliana nell'edizione del I950 per i tipi di The Reprint Society.

57. È la stessa Rosselli a confermare il suo interesse per l'autore inglese in un'intervista rilasciata a una giovane studentessa barese nel 1990: si veda Rossana Inchingolo, Il corpo della poesia, Tesi discussa alla Facoltà di Lingue e letterature straniere, Bari, A.A. I990-I99I, ora in É vostra la vita che ho perso, cit., pp. I32-I37. 
poesia del gallese quanto di quella della cosmopolita Amelia e si direbbe per lei, limitatamente alle opere della fase giovanile, quanto dissero alcuni detrattori di Dylan Thomas, ovvero che si tratta di una poesia legata ad alcune tematiche elementari, nascita-copulazione ed estinzione. La sessualità, per il gallese, è un passaggio cruciale, segno di corruzione della carne (dunque collimante con la morte) ma pure di rigenerazione: dietro ci sta la passione per la psicoanalisi, non junghiana - come sarà per Amelia ma freudiana.

Affini sono l'utilizzo frequente di alcune metafore (acqua come fonte di vita e aridità come segno di morte, il simbolismo cristiano, il simbolismo sessuale), il frequente accostamento di aggettivi astratti e sostantivi concreti, le tante immagini di corporeità, il largo impiego di formule sinestetiche. Penso in questo senso alle valenze sinestetiche della «slow moonlight» (SL 26), della "cannibal laughter» (SL IO2), la "penetrating delicacy» (SL I54), il «tender fright» (SL i54), la «brilliant thirst» (SL I56): molti versi delle poesie thomasiane mostrano ugualmente un uso continuo della sinestesia e ci sono dita che indicano (nel senso di vedono), orecchie che mormorano e vedono, narici che vedono in When all my five and country senses see, un dire che ha un colore in Once it was the colour of saying ${ }^{58}$. L'uso della sinestesia credo rifletta la comune convinzione che i sensi sono parte della mente, una sorta di corpo dell'anima, tanto che è bene avversare una concezione della poesia che separi la mente dai sensi dal momento che la distinzione stessa dei sensi è — alfine un'imposizione artificiale della razionalità. Lo scompiglio dei sensi assicura una percezione unificata del mondo esterno, per quanto non riesca a far conseguire un dominio assoluto del Tutto. Attraverso il corpo e i suoi sensi ci si protende verso le cose del mondo.

Ma già Jung sosteneva che «la vita dell'uomo è un continuo titubare tra spirituale e concreto» (PS Io4), e altro ambito lessicale di forte preponderanza è quello che contrappone la sfera della corporeità a quella della spiritualità: tra gli scaffali dell'ultima casa romana dell'autrice c'era il volume The Bhagavad-Gita, or, The Lord's Song nella traduzione del 1923 di Annie Besant per la Theosophical Press di Chicago. Il termine è un plu-

58. Uso qui l'edizione Dylan Thomas, Poesie, a cura di Renzo Crivelli con traduzione di Ariodante Marianni, Torino, Einaudi, 2002. Al Fondo Rosselli di Viterbo sono presenti i volumi di Dylan Thomas posseduti dalla Rosselli: Poesie inedite, a cura di Ariodante Marianni, Torino, Einaudi, I980; Poesie, traduzione, introduzione e note di Roberto Sanesi, Modena, Guanda, 1954; Portrait of the Artist as a Young Dog, London, Dent, 1940, rist. 1958; Quite Early One Morning: Broadcasts, preface by Aneirin Talfan Davies, Paperback ed., London, Dent \& Sons, 1967; Under Milk Wood: A Play for Voices, preface by Daniel Jones, London, Dent \& Sons, 1962, rist. 1974. Tutti i volumi presentano tracce di lettura e annotazioni a matita o a penna. 
rale femminile sanscrito e corrisponde al titolo di un poema di contenuto religioso, che ha assunto il valore di testo sacro dell'Induismo: Krishna, incarnazione di Dio e identificabile con l'Ātman, ovvero il proprio Sé più profondo e immortale, indica ad Arjuna le tecniche mistiche (Yoga) per liberarsi definitivamente dal ciclo delle nascite e delle morti (samsāara) e ottenere la liberazione (mokșa). Il volume di Amelia mostra evidenti tracce di lettura e frequenti note, come quella a pastello blu in margine al pensiero «(20)», a lato del quale aggiunge con una penna nera la dicitura «The Spirit»:

He is not born, nor doth he die; nor having been, ceaseth he any more to be; unborn, perpetual, eternal and ancient, he is not slain when the body is slaughtered.

Nel Diario già si leggeva che «the sensorial and the spiritual [...] both have their centers, at all points of the turning world» (PS I22). L'apertura della raccolta inglese è all'insegna della decapitazione di cui dicevo, con in scena due "tenere grasse pesanti mani», delle "anime svestite d'un corpo»e del sangue che imporpora tutto, ma in un quadro nel quale aleggia anche «la fragranza fresca e dolce dell'incenso». Del resto altrove la Rosselli parla di una mistica ascesi il cui esito è però una luna sordida (SL 23): ancora una volta l'antinomia di corpo e spirito ridotta a unità.

\section{Postilla imperfetta}

La lettura intima e costante di Amelia Rosselli, così come si intuisce da questi miei «appunti sparsi e persi» 59 , insegna che quello che si può isolare sono solo affinità elettive trasversali, che travalicano il confinamento a un supposto settore di scrittura femminile: ci si trova spesso coinvolti in un gioco di rifrazioni in una realtà dove non sempre ci sono ipotesti e contraffazioni, ma - spesso - semplicemente epifanie poetiche che si manifestano in modi simili, disseminando semmai tracce mnestiche letterarie qua e là, come si è visto per la Woolf e Thomas. Uno sguardo orientato da un'ipotesi 'di genere' si troverebbe forse deluso, per quanto la produzione nella lingua di 'genere' femminile del patrimonio genetico della poetessa, ovvero l'inglese, si consente maggiori varchi verso i campi della mistica, della teatralità e della psicanalisi, usando la lingua per scavare dentro di sé.

59. Mutuo un'espressione rosselliana: si veda Amelia Rosselli, Appunti sparsi e persi (I966-I977), Roma, Empiria, 1998. 
Per la Rosselli si deve concludere quanto lei concluse per Sylvia Plath, ovvero che «non è mai stucchevolmente femminile, non rappresenta mai l'anima femminile come genere in sé. [...] Ha una sua virilità femminile. Stilisticamente è un poeta a sé, non si può dire da chi derivi» ${ }^{6}{ }^{\circ}$. Oltretutto, 'sorellanze' a parte, l'indagine che suggerisco dimostrerebbe che la trasversalità attraversa anche territori di marca maschile, si incrocia frequentemente con Shakespeare e passa dall'altra parte del letto: l'unica matrice comune che credo si possa isolare, l'unica marca che accomuna tutte le voci che si incontrano, è quella che vorrei chiamare dell'eresia, cifra stilistica e poetica che si oppone all'esistente (è, in questo senso, anti-istituzionale), accetta la sperimentazione, vive la lingua come cosa estremamente seria e visceralmente sentita per esperire il mondo.

\section{Bibliografia essenziale}

Amelia Rosselli, "Galleria», numero speciale a cura di Daniela Attanasio ed Emmanuela Tandello, 48, I-2, gennaio-agosto 1997.

Amelia Rosselli. Un'apolide alla ricerca del linguaggio universale, a cura di Stefano Giovannuzzi, Atti della giornata di studio, Firenze, Gabinetto Vieusseux, 29 maggio 1998, "Quaderni del Circolo Rosselli», Firenze, Giunti, 17, 1999.

Anzieu Didier, Il pensare. Dall'io-pelle all'io-pensante, Roma, Borla, 1996 (edizione originale 1994).

Baudrillard Jean, L'altro visto da sé, Genova, Costa \& Nolan, 1997 (edizione originale 1987).

Bisanti Tatiana, La poesia di Amelia Rosselli: il silenzio disturbato, la comunicazione assoluta, in Scrittura femminile, Italienische Autorinnen in 20. Jahrhundert zwischen Historie, Fiktion und Autobiographie, a cura di Irmgard Scharold, Tübingen, Gunter Narr Verlag, 2002, pp. 26I-280. Caporali Marco, Intervista ad Amelia Rosselli, "Poesia», aprile I990, p. 9. Cavarero Adriana, Nonostante Platone: figure femminili nella filosofia antica, Roma, Editori Riuniti, I999.

Cixous Hélène, Entre l'écriture, Paris, Des Femmes, 1986.

—, Il teatro del cuore, Parma, Pratiche, 1992 (edizione originale 1990).

Dolce Ambrogio, Amelia Rosselli: poesia non necessariamente ascientifica (intervista), «Idea», gennaio-febbraio I988, p. 42.

6o. Marco Caporali, Donne che traducono donne: Amelia Rosselli e Sylvia Plath, in La furia dei venti contrari, cit., p. 243 (già in «Reporter», 22 novembre 1985 , p. 2). 
Fotobiografia: Conversazione con Aldo Rosselli, a cura di Siriana Sgavicchia, in Dossier Amelia Rosselli, "Il Caffè Illustrato», I3-I4, luglio-ottobre 2003, pp. 64-7I.

Frabotta Biancamaria, Donne in poesia, Roma, Savelli, 1976.

Jung Carl Gustav, Opere complete, Torino, Bollati Boringhieri, I970-.

La Penna Daniela, "Cercatemi e fuoriuscite": Biography, Textuality, and Gender in Recent Criticism on Amelia Rosselli, «Italian Studies», 65, 2, July 20IO, pp. 278-285.

Lorenzini Niva, La poesia italiana del Novecento, Bologna, Il Mulino, 1999.

Moe Nelson, At the Margins of Dominion: The Poetry of Amelia Rosselli, «Italica», 69, 2, Summer 1992, pp. 177-195.

Moroni Mario, La presenza complessa: identità e soggettività nelle poetiche del Novecento, Ravenna, Longo, 1998.

Pasolini Pier Paolo, Notizia su Amelia Rosselli, «Il Menabò», 6, I963, pp. 66-69.

Perrella Silvio, Per Amelia Rosselli, «Nuovi argomenti», nuova serie I2, I997, pp. I2-I3.

Pieri Paolo Francesco, Dizionario junghiano, Torino, Bollati Boringhieri, I998.

Rosselli Amelia, Istinto di morte e istinto di piacere, "Nuovi argomenti», 67-68, luglio-dicembre 1980, pp. 175-178.

—, Le poesie, a cura di Emmanuela Tandello, prefazione di Giovanni Giudici, Milano, Garzanti, 1997.

—, Sleep. Poesie in inglese, a cura di Emmanuela Tandello, Milano, Garzanti, 1992.

-, Una scrittura plurale. Saggi e interventi critici, a cura di Francesca Caputo, Novara, Interlinea, 2004.

- E Eostra la vita che ho perso. Conversazioni e interviste 1964-I995, a cura di Silvia De March e Monica Venturini, Firenze, Le Lettere, 20 Io.

-, La furia dei venti contrari, Variazioni di Amelia Rosselli. Con testi inediti e dispersi dell'autrice, a cura di Rosaria Lo Russo e Stella Savino, Firenze, Le Lettere, 2008.

Stanford Friedman Susan, Penelope's Web: Gender, Modernity, H.D.'s Fiction, Cambridge, Cambridge University Press, 1990.

TAndello Emmanuela, Amelia Rosselli, La fanciulla e l'Infinito, Roma, Donzelli, 2007.

Zambrano María, All'ombra del dio sconosciuto: Antigone, Eloisa, Dioti$m a$, a cura e con traduzione di Elena Laurenzi, Milano, Nuove Pratiche Editrice, 1997. 
Zambrano María, Filosofia e poesia, a cura di Pina De Luca, Bologna, Pendragon, 1988.

—, Verso un sapere dell'anima, Milano, Raffaelo Cortina, 1996 (edizione originale 1993). 